TO APPEAR IN The Astrophysical Journal.

Preprint typeset using LATEX style emulateapj v. 26/01/00

\title{
CONSTRAINTS ON THE STAR FORMATION RATE IN ACTIVE GALAXIES
}

\author{
MinJin Kim \\ Astronomy Program, SEES, Seoul National University, Seoul 151-742, Korea \\ LUIS C. Ho \\ The Observatories of the Carnegie Institution of Washington, 813 Santa Barbara St., Pasadena, CA 91101 \\ MYUNGSHIN IM \\ Astronomy Program, SEES, Seoul National University, Seoul 151-742, Korea
}

To Appear in The Astrophysical Journal.

\begin{abstract}
The [O II] $\lambda 3727$ emission line is often used as an indicator of star formation rate in extragalactic surveys, and it can be an equally effective tracer of star formation in systems containing luminous active galactic nuclei (AGNs). In order to investigate the ongoing star formation rate of the host galaxies of AGNs, we measured the strength of [O II] and other optical emission lines from a large sample ( 3600) of broad-line (Type 1) AGNs selected from the Sloan Digital Sky Survey. We performed a set of photoionization calculations to help evaluate the relative contribution of stellar and nonstellar photoionization to the observed strength of [O II]. Consistent with the recent study of Ho (2005), we find that the observed [O II] emission can be explained entirely by photoionization from the AGN itself, with little or no additional contribution from H II regions. This indicates that the host galaxies of Type 1 AGNs experience very modest star formation concurrent with the optically active phase of the nucleus. By contrast, we show that the sample of "Type 2" quasars selected from the Sloan Digital Sky Survey does exhibit substantially stronger [O II] emission consistent with an elevated level of star formation, a result that presents a challenge to the simplest form of the AGN unification model.
\end{abstract}

Subject headings: galaxies: active — galaxies: nuclei — galaxies: Seyfert — galaxies: starburst — quasars: general

\section{INTRODUCTION}

It is now almost universally accepted that massive black holes are common and that they play an important role in many facets of galaxy evolution (see reviews in Ho 2004). Numerous theoretical models have been proposed to explain the strong observed correlations between black hole mass and bulge properties (Magorrian et al. 1998; Gebhardt et al. 2000; Ferrarese \& Merritt 2000). While everyone agrees that the growth of black holes must be closely linked with galaxy formation (e.g., Silk \& Rees 1998; Kauffmann \& Haehnelt 2000; Begelman \& Nath 2005; Robertson et al. 2006), there is no consensus as to exactly how the two very different processes involved-accretion and star formation - are really coupled. Are they well synchronized, or does one process precede the other, and if so, what is the time lag? When the black hole is actively growing, does the feedback from the active galactic nucleus (AGN) actually trigger or inhibit star formation in the host galaxy? These important issues are unlikely to be settled through theoretical speculations or numerical simulations alone. Some empirical guidance from observations would be welcomed.

In recent years, there has been mounting evidence that AGN activity and starburst activity are often intermixed. The most commonly cited example occurs in ultraluminous infrared galaxies, whose dominant energy source has been much debated (e.g., Genzel et al. 1998). Detailed studies of individual active galaxies have revealed a significant population of young stars in a number of instances, either through stellar absorption features (e.g., Boisson et al. 2000; Canalizo \& Stockton 2000; Cid Fernandes et al. 2001) or colors (e.g., Jahnke et al. 2004).
Additional hints for a close connection between star formation and black hole accretion comes from the high degree of chemical enrichment in quasars as deduced from analysis of their spectra (Hamann et al. 2004 and references therein). Lastly, the host galaxies of quasars, at least in the nearby Universe, possess a rich supply of both molecular gas (Scoville et al. 2003) and dust (Haas et al. 2003), and thus have the potential for sustaining ongoing or future star formation.

While there is little doubt that the above observations indicate that AGN activity and star formation do often coincide within the same galaxy, one of the main challenges in interpreting this evidence in a physical context lies in the difficulty of establishing a direct, causal connection between the two phenomena. An important step forward was recently achieved. From an analysis of a large sample of narrow-line (Type 2) AGNs selected from the Sloan Digital Sky Survey (SDSS; York et al. 2000), Kauffmann et al. (2003) showed that, unlike lowluminosity sources (Ho et al. 2003), more powerful AGNs often exhibit stellar absorption features indicative of young to intermediate-age stars. Although more difficult to ascertain due to the strong contamination from the bright nucleus, Kauffmann et al. show that the spectral signatures for young stars seem to persist even among broad-line (Type 1) AGNs. ${ }^{1}$ Most crucially, they find, for their primary sample of Type 2 objects, that the fraction of young stars in the central region of the host galaxies increases with increasing AGN luminosity, providing, for the first time, tantalizing evidence for a causal link between star formation and accretion.

It is important to recognize, however, that the SDSS results constrain only the post-starburst population, with ages

\footnotetext{
${ }^{1}$ We will use the terms "Type 1" and "Type 2" AGNs to refer to broad-line and narrow-line objects, respectively. Whenever possible, we will refrain from using the terms "Seyferts" or "quasars," which, for historical reasons, carry artificial luminosity criteria.
} 
$\sim 10^{8}-10^{9}$ yr. There is no information on the presence of younger, ionizing stars (ages $\lesssim 10^{7} \mathrm{yr}$ ). Since the Type 2 AGN sample was identified using standard optical emission-line ratios designed to isolate accretion-dominated sources, by selection it excludes any sources with a sizable contribution from $\mathrm{H}$ II regions. On the other hand, it is clearly of significance to constrain the ongoing star formation rate (SFR) in AGNs, in order to assess the extent to which the two processes are coeval. AGN lifetimes are uncertain, but current best estimates range from $10^{6}$ to $10^{8} \mathrm{yr}$ (Martini 2004), precisely in the unexplored regime of interest.

Estimating SFRs in AGNs presents significant complications, since the AGN, by definition, dominates the integrated output of the source. Nearly all of the standard measures of SFRs employed for inactive galaxies (e.g., Kennicutt 1998) are rendered useless by emission from the AGN itself. There is, however, one important exception: the [O II] $\lambda 3727$ line. As discussed by Ho (2005), [O II], an emission line commonly used in spectroscopic surveys to estimate SFRs in galaxies at redshifts $z \gtrsim 0.4$, should be an equally effective tracer of H II regions in Seyferts and quasars. In such high-ionization active galaxies, the [O II] emission intrinsic to the narrow-line region (NLR) of the AGN is both observed and predicted to be low (e.g., Ferland \& Osterbrock 1986; Ho et al. 1993b). If highionization AGNs experience substantial levels of ongoing star formation, the integrated contribution from $\mathrm{H}$ II regions will boost the strength of the [O II] line (compared to, say, [O III] $\lambda 5007$, which can be largely ascribed to the AGN itself). From a survey of the existing literature on [O II] measurements on quasars, Ho (2005) concludes that their SFRs are surprisingly modest. More intriguingly, for a subset of low-redshift quasars with available molecular gas measurements, Ho finds that their star formation efficiencies (SFR per unit gas mass) appear to be abnormally low compared to either normal or infrared-bright galaxies. He suggests that this unusual behavior may be a manifestation of AGN feedback suppressing star formation.

The results of Ho (2005) regarding SFRs in quasars were based largely on [O II] strengths deduced from composite spectra (ensemble averages) constructed from quasar surveys. While the conclusions are statistically robust, it would be desirable to revisit this problem from actual measurements of a large, well-defined sample of individual objects. This is accomplished in this paper, where we make use of an extensive sample of Type 1 AGNs selected from the SDSS to investigate the properties not only of [O II], but also a number of other diagnostically important narrow optical emission lines. We perform a new set of photoionization calculations to demonstrate that the observed strength of [O II] emission is entirely consistent with standard AGN photoionization. These results support and strengthen the conclusion of Ho (2005) that Type 1 AGNs experience very limited ongoing star formation. By contrast, we show that Type 2 quasars exhibit markedly enhanced [O II] emission, and hence presumably elevated SFRs. This result seems to violate the basic orientation-dependent unification model for AGNs.

We adopt the following cosmological parameters: $H_{0}=$ $100 \mathrm{~h}=71 \mathrm{~km} \mathrm{~s}^{-1} \mathrm{Mpc}^{-1}, \Omega_{\mathrm{m}}=0.27$, and $\Omega_{\Lambda}=0.75$ (Spergel et al. 2003).

\section{SAMPLE SELECTION}

Our goal is to investigate the narrow-line spectrum of a large, homogeneous sample of broad-line (Type 1) AGNs. We utilize the database from SDSS (Stoughton et al. 2002; Strauss

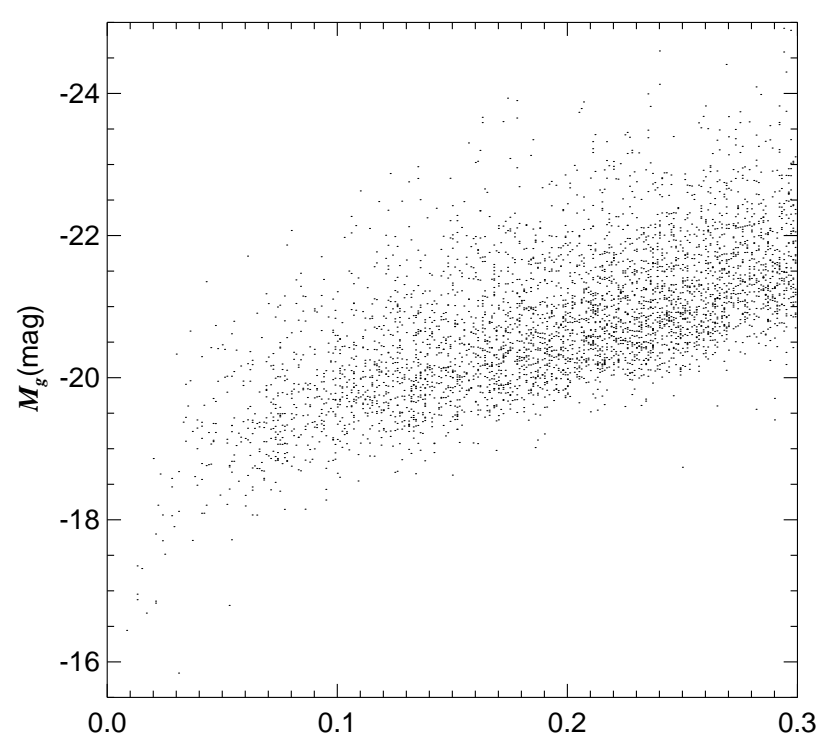

FIG. 1.- The distribution of $g$-band azbsolute magnitudes as a function of redshift for the sample. The magnitudes are "PSF" magnitudes, as defined by SDSS, and they have been corrected for Galactic extinction.

et al. 2002), whose spectra (taken with $3^{\prime \prime}$-diameter fibers) cover $\sim 3800-9200 \AA$ with an instrumental resolution of $\lambda / \Delta \lambda \approx 1800-2100$. This paper makes use of data from the Third Data Release (DR3) of SDSS (Abazajian et al. 2005), from which we have selected a total of 4086 objects flagged by the spectroscopic pipeline as "AGN" that have a redshift confidence larger than 0.9 and a redshift $z<0.3$. The redshift cut ensures that the diagnostically important [S II] $\lambda \lambda 6716,6731$ doublet falls comfortably within the bandpass. After inspection of the spectra, we discarded 498 that either had gaps in the spectral coverage or had at least one of the main emission lines corrupted, leaving a final sample of 3588 objects. Figure 1 shows the distribution of absolute ("PSF") $g$-band magnitudes as a function of redshift. The magnitudes were corrected for Galactic extinction, using the extinction values of Schlegel et al. (1998) and the extinction curve of Cardelli et al. (1989), but we did not apply a K-correction because of the ambiguity introduced by the uncertain amount of galaxy contamination. Since local field galaxies have an absolute $g$-band magnitude of $M_{*}=-20.44-5 \log h \approx-21.2$ (Blanton et al. 2003), it is clear that some of our objects are significantly contaminated by host galaxy emission. We simulated the expected magnitude due to K-correction by adding different relative fractions of quasar and galaxy light, which we approximate, respectively, using a composite quasar spectrum (Vanden Berk et al. 2001) and an early-type galaxy template (Coleman et al. 1980). For an AGN contribution of $40 \%$ or more at $5100 \AA$, we find that the expected K-correction is less than $0.4 \mathrm{mag}$. Since the exact absolute magnitudes are not critical for our study, we will neglect it hereafter.

\section{SPECTRAL FITTING}

The spectra of Type 1 AGNs are generally very complex. Figure 2 illustrates some spectra from our sample. While in this study we are primarily interested in the narrow-line spectrum, which is somewhat easier to measure, we will describe in greater detail our method for analyzing the entire spectrum. We illustrate an example of spectral fitting in Figure 3. After re- 
moving the Galactic extinction, the spectrum is transformed to the restframe using the redshift measured by the SDSS pipeline. A full decomposition of the continuum spectrum requires fitting of four components. First, we allow for the presence of a galaxy component. As mentioned in $\S 2$, we expect galaxy contamination to be important in the lower-luminosity objects in our sample. While more sophisticated methods of galaxy modeling have been attempted (e.g., Ho et al. 1993a, 1997a; Greene \& Ho 2004), Greene \& Ho (2005b) find that a simple scaling and subtraction of a velocity-broadened K-giant star are usually sufficient for Type 1 AGNs with the signal-to-noise ratio (S/N) of SDSS. As in Greene \& Ho (2005b), we use the equivalent width (EW) of the Ca II K $\lambda 3934$ absorption line as a guide to the level of galaxy contamination and how much starlight to subtract. These authors find that an EW of $1.5 \AA$ for Ca II K corresponds to a galaxy contribution of $\sim 10 \%$. We omit the K-giant component for EW(Ca II K) $<1.5 \AA$, and we scale it appropriate for EWs larger than this value. Second, the ultraviolet and optical continuum contains a prominent featureless, nonstellar component, which previous studies (e.g., Francis et al. 1991; Vanden Berk et al. 2001) have approximated by a double power law, with a spectral break at $\sim 5000 \AA$. We find, however, that after accounting for the galaxy component the second, long-wavelength power-law component is usually unnecessary, and thus we model the featureless continuum using a single power law. Third, following Grandi (1982), we include the contribution from a Balmer continuum, assuming that it is optically thick and emitted by a uniform temperature of $T_{e}=15,000 \mathrm{~K}$; we do not attempt to model the higher-order Balmer lines. Lastly, we model the "pseudo-continuum" generated by the plethora of broad and blended Fe II multiplets, which, for our present application, are particularly troublesome for the region containing the $\mathrm{H} \beta$ and [O III] emission lines. Following standard practice (Boroson \& Green 1992), we remove the Fe blends by scaling and broadening an Fe template generated from the spectrum of $\mathrm{I} Z \mathrm{~W} 1$, kindly provided by $\mathrm{T}$. A. Boroson. (The region between $3082 \AA$ and $3685 \AA$ is not modeled because of a gap in the current Fe template.) After subtracting the galaxy component, we simultaneously fit the quasar spectrum with the power-law continuum, Balmer continuum, and Fe template. We perform the fit over the following regions, which are devoid of strong emission lines: 3550-3645, 41704260, 4430-4770, 5080-5550, 6050-6200, and 6890-7010 Å.

With a pure emission-line spectrum at hand, the next task is to fit the lines (see bottom of Fig. 3). Since the continuum near $\sim 3700 \AA$ is still not completely modeled (we neglected higherorder Balmer lines in the Balmer continuum model and the $\mathrm{Fe}$ template is incomplete), we have decided to fit [O II] $\lambda 3727$ with a single Gaussian above a locally defined continuum. This procedure, although crude, should be adequate for our purposes. We note that Greene \& Ho (2005a) find that [O II], at the $\mathrm{S} / \mathrm{N}$ of SDSS, is usually well-fitted by a single Gaussian. However, because of the strong confusion with the broad-line region, the lines around $\mathrm{H} \alpha$ and $\mathrm{H} \beta$ - and especially the narrow components of these lines themselves-require more complex treatment. Previous studies (Ho et al. 1997b; Greene \& Ho 2004, $2005 \mathrm{~b}$ ) find that the $[\mathrm{S} \mathrm{II}] \lambda \lambda 6716,6731$ doublet serves as a very effective template to model [N II] $\lambda \lambda 6548,6583$ and the narrow component of $\mathrm{H} \alpha$. In objects with sufficiently strong [S II] (S/N $\gtrsim 3$ ), we fit each of the [S II] lines with a multi-Gaussian model (usually just two suffice), holding fixed the known separation of the two lines. This [S II] template is then used as a model for [N II] and narrow $\mathrm{H} \alpha$. The separation of the [N II] doublet is held fixed, as is its flux ratio (2.96). In practice, we often find it necessary to also fix the position of the $\mathrm{H} \alpha$ component relative to [N II]. In cases where [S II] has $\mathrm{S} / \mathrm{N} \lesssim 3$, we have no alternative but to use [O III] $\lambda 5007$ as a template, even though we know that this is not entirely adequate because [O III] often has a blue, asymmetric wing that is generally absent from [S II] (Greene \& Ho 2005a). As in Greene \& Ho (2005a, 2005b), we model each of the [O III] $\lambda \lambda 4959,5007$ lines with a twocomponent Gaussian, and we constrain the narrow component of $\mathrm{H} \beta$ to the narrow component of $\mathrm{H} \alpha$, keeping the flux ratio fixed to the Case $\mathrm{B}^{\prime}$ value of $\mathrm{H} \alpha / \mathrm{H} \beta=3.1$ (Osterbrock 1989). ${ }^{2}$ The broad components of $\mathrm{H} \alpha$ and $\mathrm{H} \beta$ are fitted with multiple Gaussian components.

The vast majority ( $\sim 95 \%$ ) of our sample yielded unambiguous detection of the strongest emission lines considered in this study ([O II], [O III], $\mathrm{H} \alpha$, and [N II]). The success rate, however, was lower for $\mathrm{H} \beta$ (66\%), and it was disappointingly low (44\%) for [O I] and [S II], which, in addition to being weaker, also lie in the reddest portion of the SDSS bandpass, where the effective $\mathrm{S} / \mathrm{N}$ is decreased by systematic noise due to imperfect removal of night-sky emission, telluric absorption, and detector fringing. We set a $3 \sigma$ upper limit for the nondetections by assuming that the line has a Gaussian profile equal to that of the average of the detected narrow lines and an amplitude 3 times the local rms of the local continuum.

\section{RESULTS}

\section{1. [O II] and [O III] Luminosities}

Figure 4 shows the distributions of [O II] $\lambda 3727$ and [O III] $\lambda 5007$ luminosities for our sample. The [O II] luminosities range from $\sim 10^{40}$ to $3 \times 10^{42} \mathrm{ergs} \mathrm{s}^{-1}$, with an average of $\left.\left\langle L_{[\mathrm{O}} \mathrm{II}\right]\right\rangle=5.2 \times 10^{40} \mathrm{ergs} \mathrm{s}^{-1}$. For comparison, we overplot the sample of Type 2 AGNs studied by Kauffmann et al. (2003), which, in terms of narrow-line emission, is approximately a factor of 2 less luminous than our sample. As discussed in Ho (2005), this level of [O II] emission, taking conservative assumptions about AGN contamination and corrections for extinction and metallicity, corresponds to SFRs of only $\sim 0.5 M_{\odot}$ $\mathrm{yr}^{-1}$. Figure 5 shows the luminosity of [O III] plotted versus the line ratio $[\mathrm{O} \mathrm{III}] /[\mathrm{O} \mathrm{III}]$. The $[\mathrm{O} \mathrm{II}] /[\mathrm{O} \mathrm{III}]$ ratio spans a wide range, in the extreme by nearly 2 orders of magnitude from $\sim 0.06$ to $\sim 5$, but most cluster around $\sim 0.2-1$. Assuming that [O III] is produced entirely by the AGN and is a good tracer of AGN power (e.g., Kauffmann et al. 2003), it is clear that the $[\mathrm{O} \mathrm{II}] /[\mathrm{O} \mathrm{III}]$ ratio decreases strongly with increasing [O III] strength. For comparison, we also overplot the sample of Kauffmann et al. (2003) to illustrate that the lower-luminosity Type 2 objects also obey roughly the same pattern. This trend is easy to explain in the context of AGN photoionization models, in which the $[\mathrm{O} \mathrm{II}] /[\mathrm{O} \mathrm{III}]$ ratio varies strongly as a function of

\footnotetext{
${ }^{2}$ Note that this constraint effectively assumes that the NLR experiences negligible internal extinction. We have experimented with leaving free the amplitude of the narrow component of $\mathrm{H} \beta$. The resulting distribution of Balmer decrement is sharply peaked at a value of $\mathrm{H} \alpha / \mathrm{H} \beta=3.3$, suggesting that any internal extinction is evidently quite modest $\left(A_{V} \approx 0.2 \mathrm{mag}\right)$. However, a minority of the objects have extremely tiny, unphysical $\mathrm{H} \alpha / \mathrm{H} \beta$ ratios, resulting from severe overestimation of the $\mathrm{H} \beta$ strength. Since the majority of the objects seem to be well characterized by small amounts of internal extinction in their NLR, we have decided that it would be prudent to fix the $\mathrm{H} \alpha / \mathrm{H} \beta$ ratio to the Case $\mathrm{B}^{\prime}$ value. As an aside, it is interesting, and probably not coincidental, that Greene \& Ho (2005b) find that the Balmer decrements of the broad-line region in their sample of SDSS Type 1 AGNs also indicate little, if any, internal extinction along the line of sight.
} 


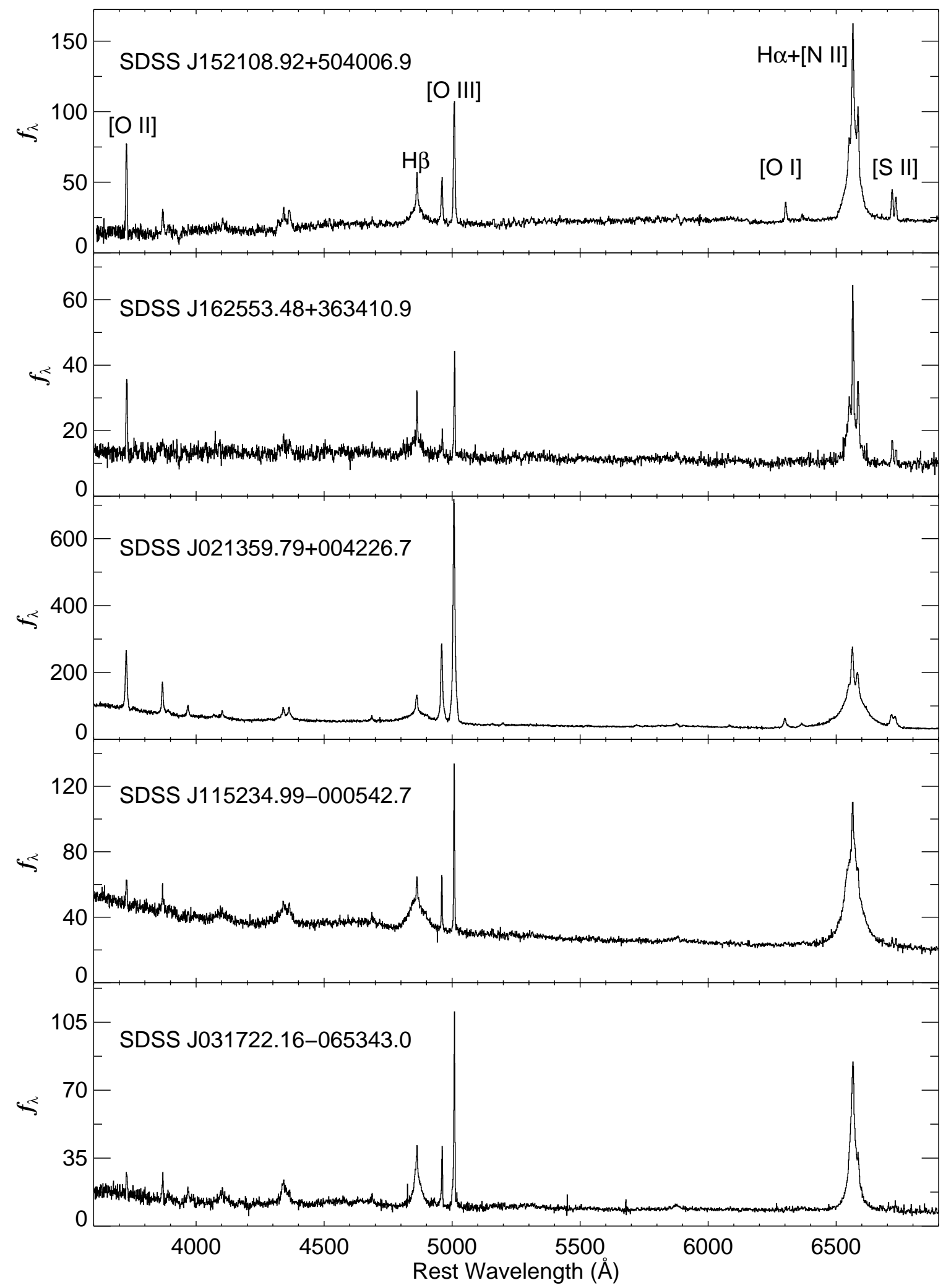

FIG. 2.- Sample spectra of our objects, chosen to span a range in S/N and [O II] strength. The restframe flux, $f_{\lambda}=f_{\lambda, \text { obs }}(1+z)^{3}$, is in units of $10^{-17}$ ergs s $\mathrm{s}^{-1}$ $\mathrm{cm}^{-2} \AA^{-1}$. 

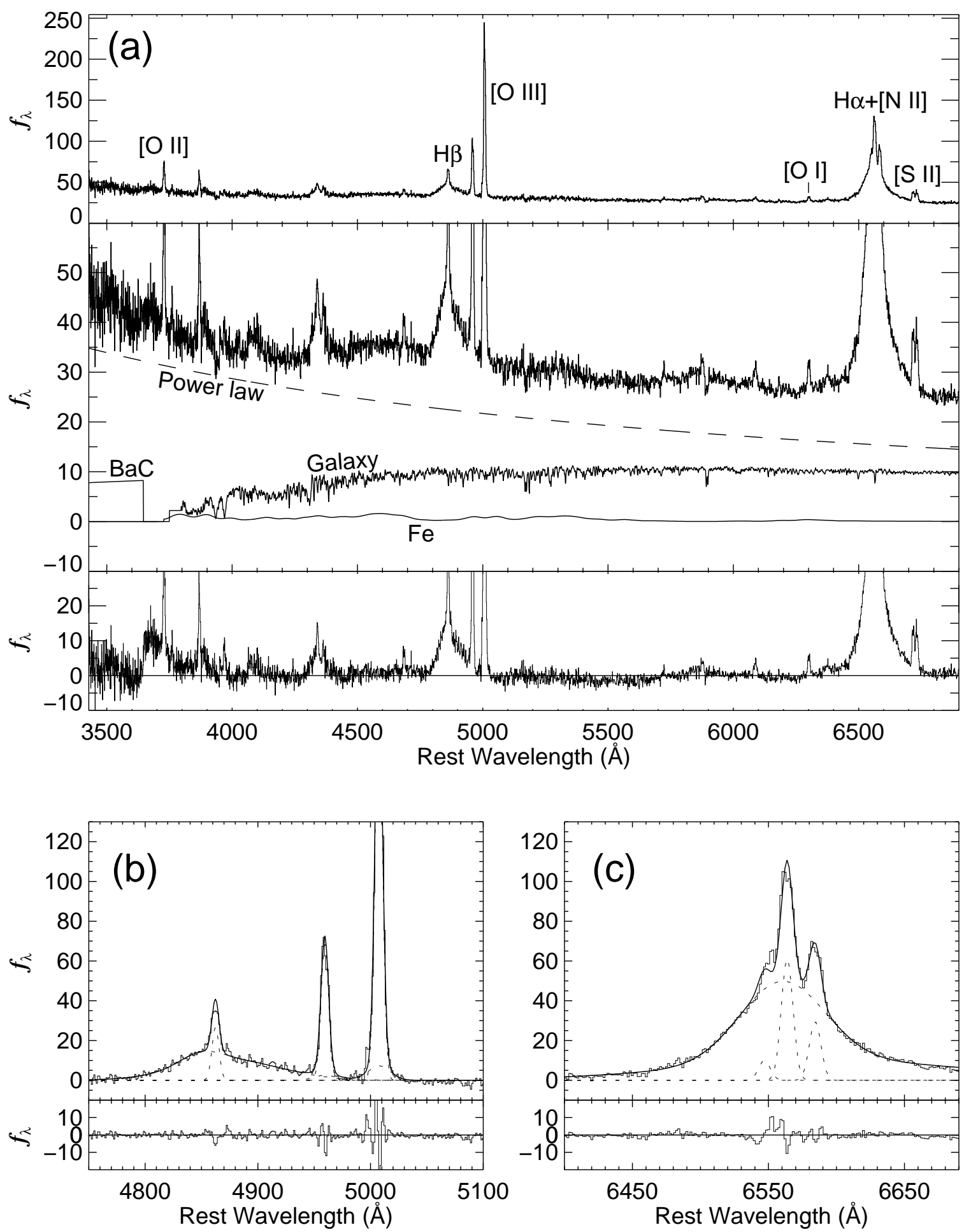

FIG. 3. - Example of spectral decomposition for one of the sample objects (SDSS J161141.95+495847.9). The restframe flux, $f_{\lambda}=f_{\lambda, \text { obs }}(1+z)^{3}$, is in units of $10^{-17} \mathrm{ergs} \mathrm{s}^{-1} \mathrm{~cm}^{-2} \AA^{-1}$. (a) The top panel shows the original spectrum in full scale. The middle panel gives a magnified view of the original spectrum, with some key emission lines labeled, along with the different continuum components included in the fit [featureless power law, Balmer continuum (BaC), galaxy (K-giant star), and the Fe template]. The continuum-subtracted emission-line spectrum is shown in the bottom panel. Note that the Fe template does not cover the region between 3082 and $3685 \AA$, and the $\mathrm{BaC}$ component does not properly model the higher-order Balmer lines in the region $3650-3800 \AA$, resulting in strong residuals in the continuum near [O II]. To illustrate the detailed fits to the emission lines, we show an expanded view of the regions surrounding $(b) \mathrm{H} \beta$ and [O III] $\lambda \lambda 4959$, 5007 and $(c) \mathrm{H} \alpha$ and $[\mathrm{N} \mathrm{II}] \lambda \lambda 6548,6583$. 

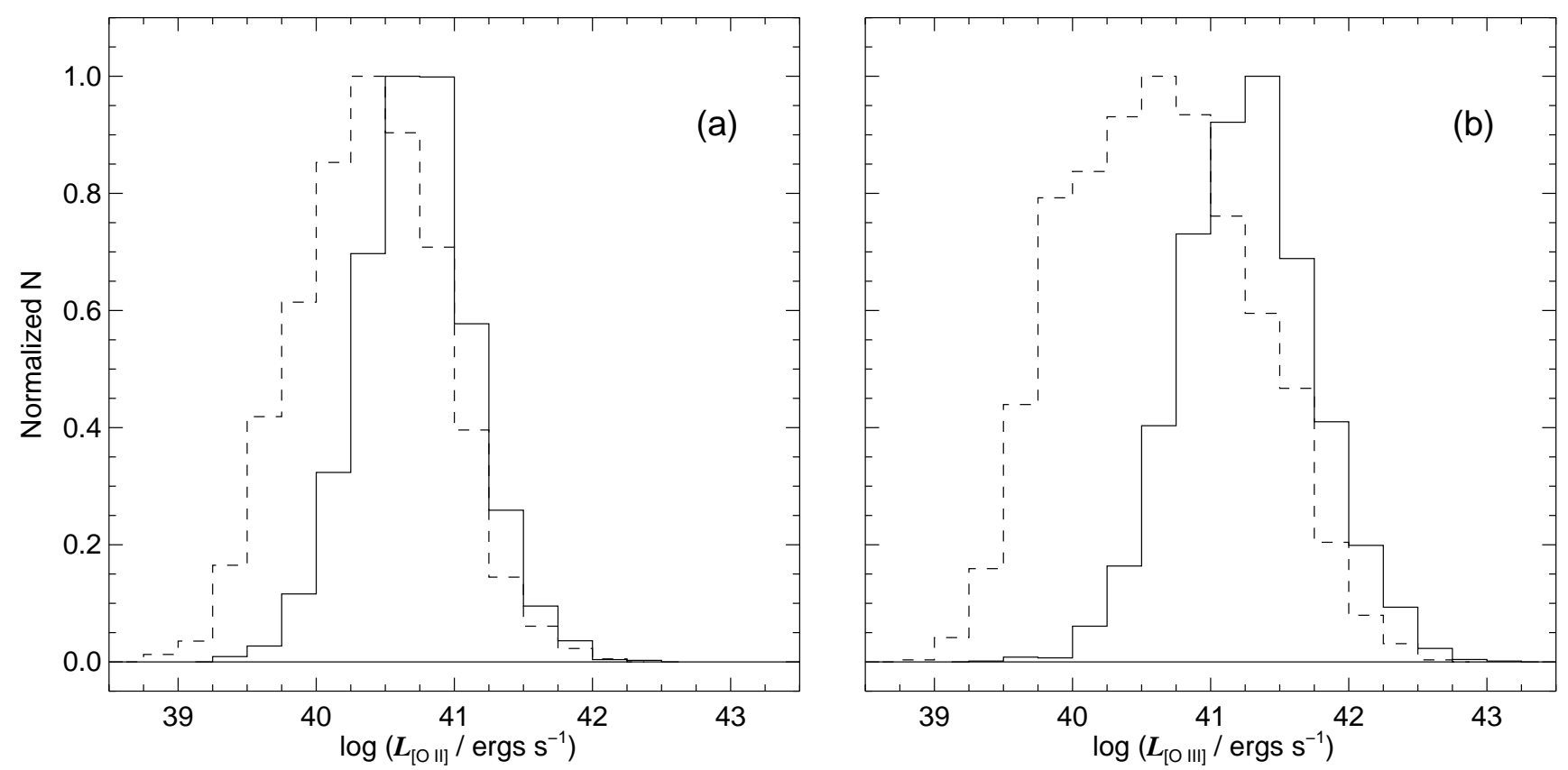

FIG. 4.- Relative distribution of (a) [O II] and (b) [O III] luminosity. The width of each bin is 0.25 dex. The solid line represents the Type 1 AGNs from our sample, and the dashed line represents the Type 2 AGNs from Kauffmann et al. (2003). The Type 2 objects have been corrected for internal extinction, as given in Kauffmann et al.

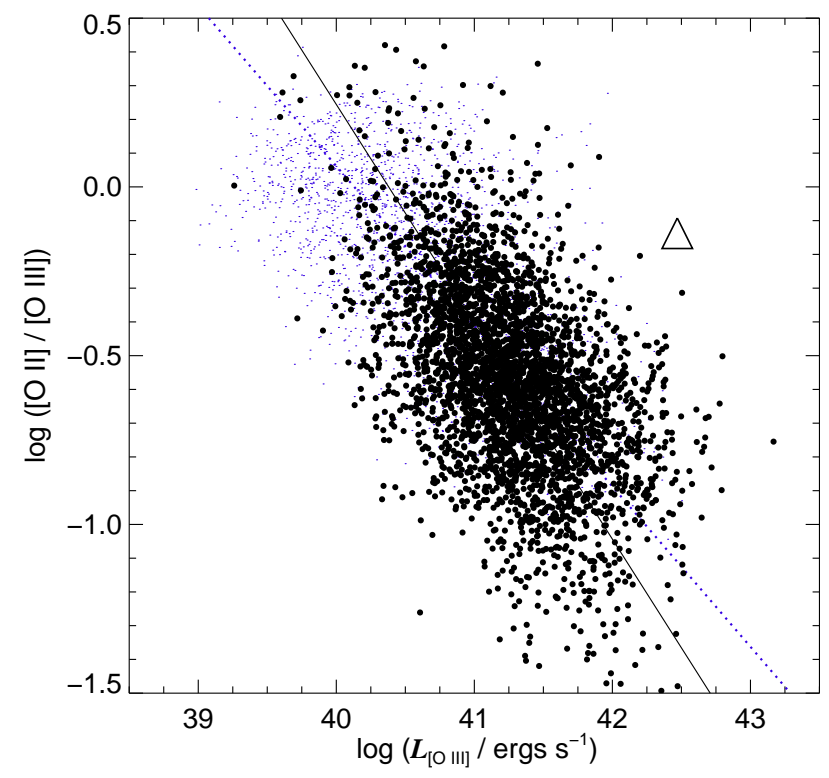

FIG. 5.- The [O II]/[O III] ratio vs. [O III] luminosity for Type 1 AGNs (large black dots) and Type 2 AGNs (small blue dots; from Kauffmann et al. 2003). The solid and dotted lines give the ordinary least-squares bisector regression for the Type 1 and Type 2 objects, respectively. The large triangle marks the location of the sample of Type 2 quasars from Zakamska et al (2003), using the average value of the [O III] luminosity and the [O II $] /[\mathrm{O}$ III] ratio measured from their composite spectrum. The values from Kauffmann et al. and Zakamska et al. have been corrected for internal extinction.

of the ionization parameter ${ }^{3}$ (see below), but it is difficult to reconcile in a picture wherein the strength of star formation increases with increasing AGN activity. In such a scenario, the
[O II]/[O III] ratio would either remain constant or rise with increasing $\left.L_{[\mathrm{O}} \mathrm{III}\right]$.

\subsection{Photoionization Model}

To better constrain the origin of the narrow-line spectrum in general, and of the [O II] line in particular, we performed a new set of photoionization calculations using the code CLOUDY (version 94.00; Ferland et al. 1998). For an optically thick cloud of a given density and elemental abundance illuminated by an input ionizing spectrum, CLOUDY solves the equations of statistical and thermal equilibrium and produces a selfconsistent model of the run of temperature and ionization as a function of depth into the cloud. For simplicity, we assume that the cloud has a uniform density, has a plane-parallel geometry, and is dust-free. We calculated a grid of models by varying three main parameters: the hydrogen density $\left(10^{2} \mathrm{~cm}^{-3} \leq n \leq\right.$ $\left.10^{6} \mathrm{~cm}^{-3}\right)$, the ionization parameter $\left(10^{-4} \leq U \leq 10^{-1}\right)$, and the shape of the ionizing continuum $(\alpha=-0.5,-1,-1.5,-2$, and $-2.5)$. The ionizing continuum has the basic shape described by Ho et al. (1993b), where the variation is parameterized by a power-law index $\alpha$ between $10 \mu \mathrm{m}$ and $50 \mathrm{keV}$. We adopt the solar abundances as given in Grevesse \& Anders (1989), with the exception of nitrogen whose abundance is increased to twice the solar value, as suggested by Storchi-Bergmann (1991).

We begin by examining our sample using the three diagrams proposed by Veilleux \& Osterbrock (1987), which have the advantage of being insensitive to extinction corrections. As can be seen in Figure 6, the majority of the sample of Type 1 objects is well covered by our grid of models, spanning the following range of parameters: $-2.0 \leq \alpha \leq-1.0,10^{-3.3} \leq U \leq 10^{-2.0}$, and $10^{2} \mathrm{~cm}^{-3} \leq n \leq 10^{4} \mathrm{~cm}^{-3}$. The density estimate is not consistent from one diagram to another because the NLR very

\footnotetext{
${ }^{3}$ The ionization parameter, denoted by $U$, is defined to be the ratio of the ionizing photon density to the density of hydrogen.
} 


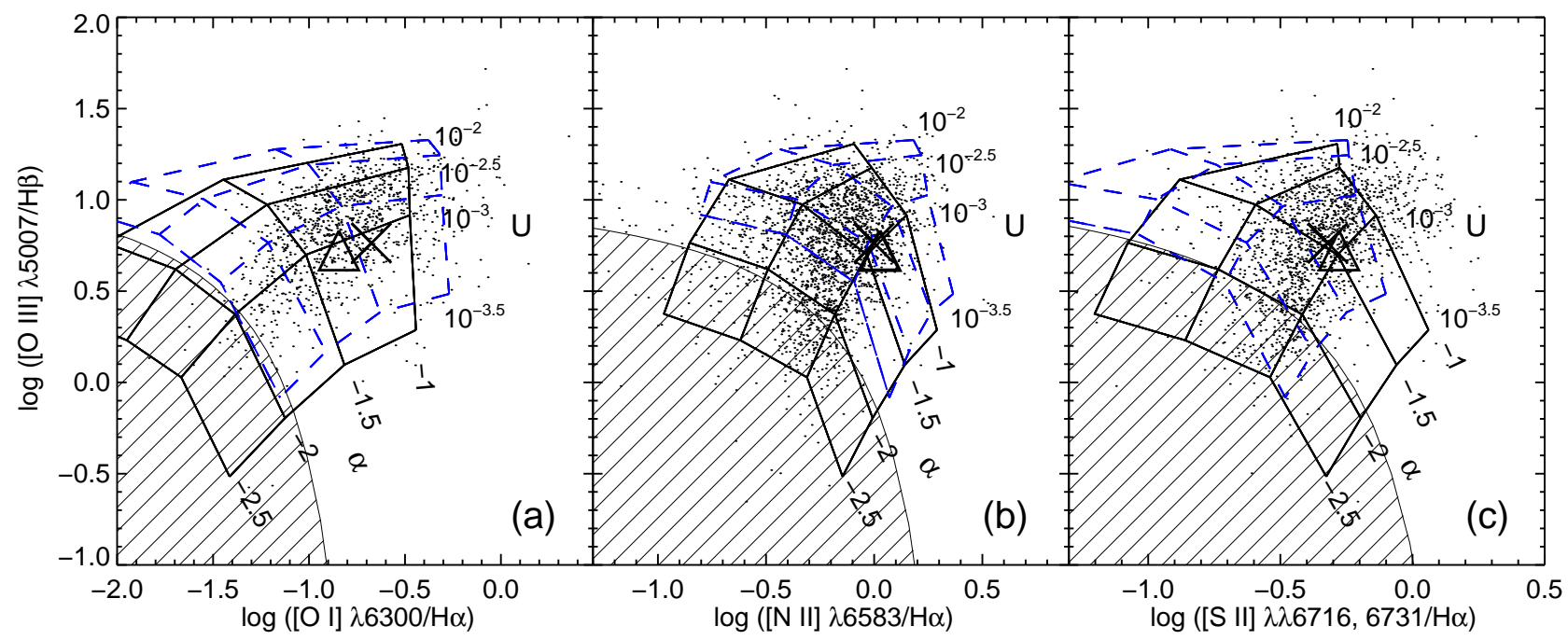

FIG. 6.- Line ratios of Type 1 AGNs (small dots) and Type 2 quasars (large triangle, from composite spectrum of Zakamska et al. 2003) compared with photoionization models. The large cross marks the location of the average value of the upper limits in our sample, which are consistent with the detections. The three Veilleux \& Osterbrock (1987) diagrams plot [O III] $\lambda 5007 / \mathrm{H} \beta$ vs. (a) [O I] $\lambda 6300 / \mathrm{H} \alpha$, (b) [N II] $\lambda 6583 / \mathrm{H} \alpha$, and (c) [S II] $\lambda \lambda 6716,6731 / \mathrm{H} \alpha$. Two grids are shown, representing $n=10^{2} \mathrm{~cm}^{-3}$ (solid black line) and $n=10^{4} \mathrm{~cm}^{-3}$ (dashed blue line). Each grid shows models for $U=10^{-2}, 10^{-2.5}, 10^{-3}$, and $10^{-3.5}$ (top to bottom) and $\alpha=-2.5,-2,-1.5$, and -1 (left to right). The shaded region represents the locus of $\mathrm{H}$ II regions, as calculated from the starburst models of Kewley et al. (2001).
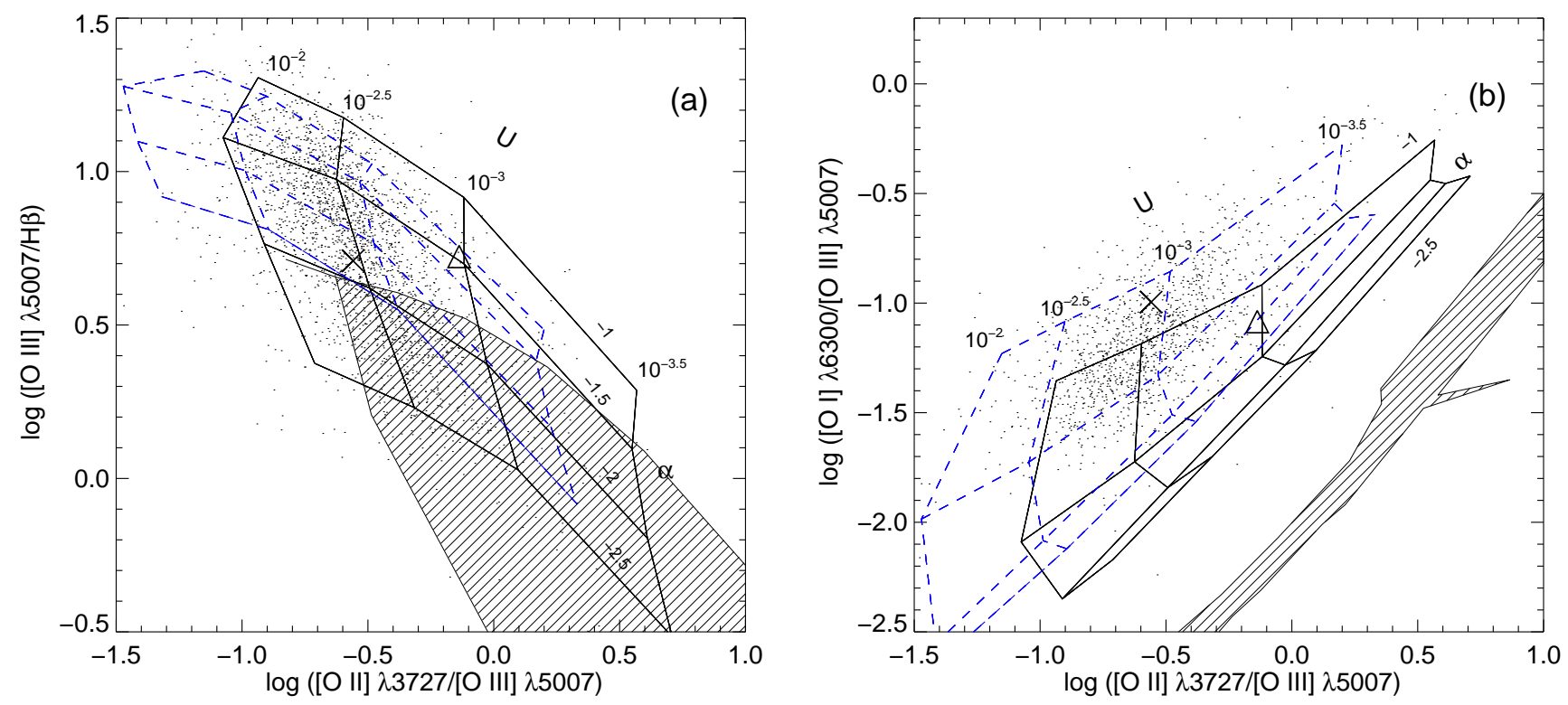

FIG. 7.- Line ratios of Type 1 AGNs (small dots) and Type 2 quasars (large triangle, from composite spectrum of Zakamska et al. 2003, with internal extinction correction applied) compared with photoionization models. The large cross marks the location of the average value of the upper limits in our sample, which are consistent with the detections. Diagnostic diagrams showing (a) [O III] $\lambda 5007 / \mathrm{H} \beta$ vs. [O II] $\lambda 3727 /[\mathrm{O}$ III] $\lambda 5007$ and $(b)[\mathrm{O}$ I] $\lambda 6300 /[\mathrm{O}$ III] $\lambda 5007$ vs. [O II] $\lambda 3727 /[\mathrm{O} \mathrm{III}] \lambda 5007$. Two grids are shown, representing $n=10^{2} \mathrm{~cm}^{-3}$ (solid black line) and $n=10^{4} \mathrm{~cm}^{-3}$ (dashed blue line). Each grid shows models for $U=10^{-2}$, $10^{-2.5}, 10^{-3}$, and $10^{-3.5}$ (left to right) and $\alpha=-2.5,-2,-1.5$, and -1 (bottom to top). The shaded region represents the locus of $\mathrm{H}$ II regions, as calculated from the starburst models of Kewley et al. (2001). 
likely has a wide range of densities, and the various forbidden transitions have different critical densities (e.g., Filippenko $\&$ Halpern 1984). Nonetheless, the above range of parameters corresponds well to previous estimates made using the same version of CLOUDY (Nagao et al. 2001).

Having established that our sample of Type 1 objects has NLR properties that can be readily accommodated using standard AGN photoionization, we next turn to two diagnostic diagrams that highlight the [O II] line. Figure 7 compares the line ratio [O II] $\lambda 3727 /[\mathrm{O}$ III] $\lambda 5007$, which is a sensitive indicator of the ionization parameter, to [O III] $\lambda 5007 / \mathrm{H} \beta$ and [O I] $\lambda 6300 /[\mathrm{O}$ III] $\lambda 5007$ (Baldwin et al. 1981; Ferland \& Netzer 1983). These diagrams have the advantage of being relatively insensitive to abundance, although [O II $] /[\mathrm{O}$ III] is affected by extinction. The basic conclusion from this figure is that the observed line ratios, and in particular the [O II] strength, can be well reproduced with AGN photoionization with roughly the same range of parameters previous deduced from the Veilleux \& Osterbrock diagrams (Fig. 6). In other words, there is no need to invoke any additional source apart from the AGN, such as star formation, to account for the observed range of [O II] emission.

To reinforce our conclusion that star formation makes a negligible contribution to the narrow-line spectrum of our objects, we superimpose on Figures 6 and 7 the theoretical photoionization models of H II regions calculated by Kewley et al. (2001). These models explore a range of input parameters meant to represent realistic conditions encountered in starburst galaxies. While the $[\mathrm{O} \mathrm{II}] /[\mathrm{O} \mathrm{III}]$ and $[\mathrm{O} \mathrm{III}] / \mathrm{H} \beta$ ratios of $\mathrm{H} \mathrm{II} \mathrm{regions} \mathrm{and}$ our sample of AGNs overlap substantially (Fig. 7a), the starburst models, even under the most extreme conditions, cannot account for the strength of the low-ionization transitions ([N II], [S II], and especially [O I]) observed in the AGNs.

\section{DISCUSSION}

\subsection{AGN Feedback}

Ho (2005; see also Ho 2006) recently proposed that the [O II] $\lambda 3727$ emission line can effectively trace ongoing star formation in the host galaxies of luminous, high-ionization AGNs such as Seyferts and quasars. The [O II] line has long been used as a SFR indicator in spectroscopic surveys of distant galaxies, but previously it had not been applied to systems containing active nuclei. From a review of the extant literature on [O II] measurements in quasars, Ho came to the conclusion that the SFRs in their host galaxies are quite modest, being no greater than $\sim 1-10 M_{\odot} \mathrm{yr}^{-1}$. This result was somewhat unexpected considering the recent evidence for there being a substantial poststarburst population in AGNs (Kauffmann et al. 2003). Perhaps even more surprising, Ho found, from a more limited sample of low-redshift quasars with available $\mathrm{CO}$ measurements, that not only are the SFRs low but that the star formation efficiencies (SFR per unit gas mass) are also low, suggesting that the AGN somehow inhibits star formation.

Apart from the small sample of low-redshift objects that had individual spectroscopic data, Ho's more general conclusions regarding the quasar population as a whole was based on published measurements of [O II] strengths derived from composite, or statistically averaged, spectra. To place these results on a more secure footing, we have undertaken a major task to homogeneously measure the [O II] line in a large sample of Type 1 AGNs. Apart from [O II], we also simultaneously measured a number of other diagnostically important narrow emission lines, and we have generated a new set of photoionization models to interpret them. Our analysis indicates that the principal narrow emission lines of our sample, including [O II], can be entirely explained by AGN photoionization assuming fairly standard parameters. There is no evidence for any excess [O II] emission arising from a separate origin, such as $\mathrm{H}$ II regions. Thus, the observed [O II] strengths can be used to set a limit on the ongoing SFR in these systems. However, as discussed by Ho, a number of uncertainties complicate the SFR estimates based on the [O II] line. These include the unknown magnitude of dust extinction, the possible influence of metallicity corrections, and precisely how much of the observed [O II] strength to attribute to star formation. For illustrative purposes, we make three simple assumptions (see Ho 2005 for a more detailed discussion): (1) that the amount of dust extinction in AGN host galaxies is comparable to that deduced for moderately actively star-forming galaxies ( $A_{V} \approx 1$ mag; e.g., Hopkins et al. 2003); (2) that the metallicity is twice solar (e.g., Storchi-Bergmann et al. 1998); and (3) that one-third of the observed [O II] strength comes from H II regions. For a typical [O II] luminosity of $5.2 \times 10^{40} \mathrm{ergs} \mathrm{s}^{-1}$ (Fig. 4a), the empirical calibration of Kewley et al. (2004) yields a SFR of merely $0.5 M_{\odot} \mathrm{yr}^{-1}$. This estimate is consistent with, but even lower than, than that given by Ho (2005). To put this estimate in perspective, we note that the SFRs of nearby normal spiral galaxies, including the Milky Way, range from $\sim 1$ to $3 M_{\odot} \mathrm{yr}^{-1}$ (Solomon \& Sage 1988). The situation in AGNs is thus truly unusual.

Unlike the low-redshift quasars considered by Ho (2005), our current sample of SDSS AGNs has no available information on its cold gas content. However, the unbiased CO survey by Scoville et al. (2003) shows that optically selected quasars, not unlike those studied here, are quite gas-rich. This is not unexpected, considering that the nuclei, by selection, are quite active, and therefore accreting at a fairly high rate. Thus, although the evidence is certainly less direct, we can reasonably surmise that the objects studied here also may also possess abnormally low star formation efficiencies.

How can this finding be reconciled with Kauffmann et al.'s (2003) result that luminous AGNs show a preponderance of post-starburst activity? A possible solution is to invoke an evolutionary scenario, not unlike that proposed by Sanders et al. (1988), in which a starburst phase precedes the optically revealed quasar phase. From the age estimates given in Kauffmann et al., the time lag seems to be approximately $10^{8}$ to $10^{9}$ years. The starburst phase terminates not because all of the gas has been transformed into stars, nor because it has been completely expelled by AGN or supernova feedback, since evidently plenty of gas coexists during the quasar phase, but because the active nucleus somehow prevents the gas from forming stars. Through a combination of radiative heating and kinetic energy injection, an AGN is expected to significantly alter the thermodynamical state of the gas in the circumnuclear regions of galaxies (Begelman 1993; Di Matteo et al. 2005), but precisely how this results in suppression of star formation remains to be elucidated.

\subsection{Possible Evidence for Starburst Activity in Type 2 Quasars}

Zakamska et al. (2003) have drawn attention to a class of narrow-line AGNs from the SDSS whose [O III] line strength, when translated into continuum luminosities, place them in the league of quasars. This, along with the detection in 
Table 1. Narrow Emission-line Ratios of Type 1 AGNs and Type 2 Quasars

\begin{tabular}{lcccccc}
\hline \hline \multicolumn{1}{c}{ Group } & $\mathrm{H} \alpha / \mathrm{H} \beta$ & {$[\mathrm{O} \mathrm{II}] /[\mathrm{O} \mathrm{III}]$} & {$[\mathrm{O} \mathrm{III}] / \mathrm{H} \beta$} & {$[\mathrm{O} \mathrm{I}] / \mathrm{H} \alpha$} & {$[\mathrm{N} \mathrm{II}] / \mathrm{H} \alpha$} & {$[\mathrm{S} \mathrm{II}] / \mathrm{H} \alpha$} \\
\hline Type 1 AGNs & $3.3^{\mathrm{a}}$ & 0.27 & 6.06 & 0.16 & 0.71 & 0.44 \\
Type 2 Quasars & 4.1 & 0.75 & 5.29 & 0.14 & 1.06 & 0.58 \\
\hline
\end{tabular}

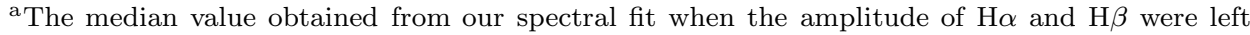
unconstrained; see $\S 3$.
}

\begin{abstract}
Note. - The line ratios for the Type 1 AGNs are the median values of the detected objects. The upper limits are consistent with these values. The line ratios for the Type 2 quasars were measured by Zakamska et al. (2003) from the composite spectrum; they have been corrected for internal extinction.
\end{abstract}

some sources of polarized broad-line emission through spectropolarimetry (Zakamska et al. 2005) and heavy photoelectric absorption through X-ray observations (Ptak et al. 2006), has prompted the identification of these objects with the long-sought "Type 2 quasars" anticipated from the the basic orientation-dependent unification model of AGNs. If the Zakamska et al. objects truly are intrinsically identical to normal, high-luminosity Type 1 AGNs, but simply viewed from a line of sight obscured from the nucleus, then the narrow-line spectrum of the two groups, assumed to be isotropic, should be similar. We were surprised to discover that this appears not to be the case. Comparison of the line strengths for our Type 1 objects with those tabulated for the composite SDSS spectrum of Zakamska et al. (2003) reveals that both groups have similar line intensity ratios, with two noticeable exceptions (see Table 1). First, the Type 2 sources have a higher Balmer decrement, and hence a higher inferred internal extinction for their NLR, compared to the Type 1 sources. Recall from our spectral fitting (\$3) that, when allowed to vary freely, the majority of our sources yielded an $\mathrm{H} \alpha / \mathrm{H} \beta$ ratio of 3.3, to be compared with $\mathrm{H} \alpha / \mathrm{H} \beta=4.1$ for the Type 2 composite. If interpreted in terms of dust extinction assuming an intrinsic Balmer decrement of 3.1 and a Galactic extinction curve, this translates into an internal extinction of $A_{V}=0.2 \mathrm{mag}$ versus $A_{V}=0.9 \mathrm{mag}$, respectively. Second, and more pertinent to the central theme of this study, we find that the Type 2 quasars exhibit an anomalously enhanced (extinction corrected) [O II]/[O III] ratio. This point is illustrated in Figures 6 and 7, where we highlight the location of the Zakamska composite in relation to the locus of the Type 1 sources. While the relatively high $[\mathrm{O}$ II $] /[\mathrm{O}$ III] ratio can normally be attributed to a somewhat lower ionization parameter, we note that this explanation is in serious conflict with the high luminosity of these sources. As discussed in $\S 4.2$, the $[\mathrm{O} \mathrm{II}] /[\mathrm{O} \mathrm{III}]$ ratio varies strongly with [O III] luminosity. For $L_{[\mathrm{O} \text { III] }} \approx 3 \times 10^{42} \mathrm{ergs} \mathrm{s}^{-1}$, the average for the Zakamska et al. sample, Figure 5 predicts that the $[\mathrm{O} \mathrm{II}] /[\mathrm{O} \mathrm{III}]$ ratio should be $\sim 0.06-0.1$. By contrast, the Type 2 quasar composite gives $[\mathrm{O} \mathrm{II}] /[\mathrm{O} \mathrm{III}]=0.75$, nearly an order of magnitude larger than the predicted value. We speculate that the enhanced [O II] emission in the Type 2 quasars comes from star formation. If the observed $[\mathrm{O} \mathrm{II}] /[\mathrm{O} \mathrm{III}]$ ratio in Type 2 quasars is $\sim 10$ times larger than expected for their [O III] luminosity, then the excess [O II] luminosity of $\sim 2 \times 10^{42} \mathrm{ergs} \mathrm{s}^{-1}$, adopting the assumptions specified in $\$ 5.1$, corresponds to an estimated SFR of $\sim 20 M_{\odot} \mathrm{yr}^{-1}$. This level of star formation qualifies as a moderately healthy starburst. Incidentally, we note that a dusty, gas-rich environment characteristic of starbursts may naturally account for the higher internal extinction mentioned above.

The possible existence of significant ongoing star formation in Type 2 quasars, in contrast to their stark absence in normal (Type 1) quasars, presents an obvious challenge to the conventional orientation-dependent model of AGN unification (Antonucci 1993). Type 2 objects are not intrinsically the same as Type 1 objects, but instead may represent an earlier evolutionary phase, perhaps similar to the scenario discussed in §5.1, wherein the central engine is either not yet fully developed or else lies buried by extended, dusty regions of star formation. In this context, the optically selected Type 2 quasars closely resemble those ultraluminous infrared galaxies that host both a strong starburst and a highly obscured AGN (e.g., Genzel et al. 1998; Gerssen et al. 2004).

Observations of radio-loud AGNs have suggested that the [O III]-emitting region, unlike that producing [O II], may not be entirely isotropic (Jackson \& Browne 1990; Hes et al. 1996). If this is generically true of all AGNs, then the [O II $] /[\mathrm{O} \mathrm{III]}$ ratio is orientation-dependent, and it may account for the higher ratios in Type 2 quasars compared to Type 1 quasars. This explanation, however, is unsatisfactory because Figure 5 shows no evidence that this pattern holds for lower-luminosity sources.

\section{SUMMARY}

We present a detailed analysis of the narrow-line spectrum of a large, homogeneous sample of broad-line (Type 1) AGNs selected from SDSS, with the primary aim of using the strength of the [O II] $\lambda 3727$ line to constrain the ongoing star formation rate in the host galaxies. Comparing the measurements with a new set of photoionization models calculated using the code CLOUDY, we find that the principal narrow optical lines, including [O II], can be readily reproduced using conventional AGN photoionization parameters. This places strong constraints on any additional starburst contribution to the [O II] strength, with resulting limits on the inferred star formation rate. Consistent with the recent study of Ho (2005), the host galaxies of Type 1 AGNs evidently experience very modest ongoing star formation. On the other hand, the sample "Type 2" quasars identified from SDSS (Zakamska et al. 2003) exhibits enhanced [O II] emission, which we argue arises from significant ongoing starburst activity. We propose an evolutionary scenario that can account for these observations. 
We would like to thank T. A. Boroson for sending us the I $\mathrm{Zw} 1$ iron template, G. J. Ferland for making available the code CLOUDY, L. J. Kewley for making available her model calculations of H II regions, and an anonymous referee for a helpful critique of our work. We are grateful to the entire SDSS collaboration for providing access to their invaluable database. M.K. has been supported in part by the BK 21 program. M.K. and
M.I. acknowledge the support from the grant No. R01-2005000-10610-0 provided by the Basic Research Program of the Korea Science \& Engineering Foundation. The work of L.C.H. was supported by the Carnegie Institution of Washington and by NASA through grants from the Space Telescope Science Institute (operated by AURA, Inc., under NASA contract NAS526555).

\section{REFERENCES}

Abazajian, K., et al. 2005, AJ, 129, 1755

Antonucci, R. 1993, ARA\&A, 31, 473

Baldwin, J. A., Phillips, M. M., \& Terlevich, R. 1981, PASP, 93, 5

Begelman, M. C. 1993, in The Environment and Evolution of Galaxies, ed. J. M. Shull \& H. A. Thronson Jr. (Dordrecht: Kluwer), 369

Begelman, M. C., \& Nath, B. B. 2005, MNRAS, 361, 1387

Blanton, M. R., et al. 2003, ApJ, 592, 819

Boisson, C., Joly, M., Moultaka, J., Pelat, D., \& Serote Roos, M. 2000, A\&A, 357,850

Boroson, T. A., \& Green, R. F. 1992, ApJS, 80, 109

Canalizo, G., \& Stockton, A. 2000, ApJ, 528, 201

Cardelli, J. A., Clayton, G. C., \& Mathis, J. S. 1989, ApJ, 345, 245

Cid Fernandes, R., Jr., Heckman, T. M., Schmitt, H. R., Golzález Delgado, R. M., \& Storchi-Bergmann, T. 2001, ApJ, 558, 81

Coleman, G. D., Wu, C.-C., \& Weedman, D. W. 1980, ApJS, 43, 393

Di Matteo, T., Springel, V., \& Hernquist, L. 2005, Nature, 433, 604

Ferland, G. J., Korista, K. T., Verner, D. A., Ferguson, J. W., Kingdon, J. B., \& Verner, E. M. 1998, PASP, 110, 761

Ferland, G. J., \& Netzer, H. 1983, ApJ, 264, 105

Ferland, G. J., \& Osterbrock, D. E. 1986, ApJ, 300, 658

Ferrarese, L., \& Merritt, D. 2000, ApJ, 539, L9

Filippenko A. V., \& Halpern, J. P. 1984, ApJ, 285, 458

Francis, P. J., Hewett, P. C., Foltz, C. B., Chaffee, F. H., Weymann, R. J., \& Morris, S. L. 1991, ApJ, 373, 465

Gebhardt, K., et al. 2000, ApJ, 539, L13

Genzel, R., et al. 1998, ApJ, 498, 579

Gerssen, J., van der Marel, R. P., Axon, D., Mihos, J. C., Hernquist, L., \& Barnes, J. E. 2004, AJ, 127, 75

Grandi, S. A. 1982, ApJ, 255, 25

Greene, J. E., \& Ho, L. C. 2004, ApJ, 610, 722

2005a, ApJ, 627, 721

. 2005b, ApJ, 630, 122

Grevesse, N., \& Anders, E. 1989, in AIP Conf. Proc. 183, Cosmic Abundances of Matter, ed. C. J. Washington (New York: AIP), 1

Haas, M., et al. 2003, A\&A, 402, 87

Haehnelt, M. G., \& Rees, M. J. 1993, MNRAS, 263, 168

Hamann, F., Dietrich, M., Sabra, B. M., \& Warner, C. 2004, in Carnegie Observatories Astrophysics Series, Vol. 4: Origin and Evolution of the Elements, ed. A. McWilliam \& M. Rauch (Cambridge: Cambridge Univ. Press), 443

Hes, R., Barthel, P. D., \& Fosbury, R. A. E. 1996, A\&A, 313, 423

Ho, L. C. 2004, ed., Carnegie Observatories Astrophysics Series, Vol. 1: Coevolution of Black Holes and Galaxies (Cambridge: Cambridge Univ. Press)

2005, ApJ, 629, 680
2006 (astro-ph/0511157)

Ho, L. C., Filippenko, A. V., \& Sargent, W. L. W. 1993a, ApJ, 417, 63

1997a, ApJS, 112, 315

. 2003, ApJ, 583, 159

Ho, L. C., Filippenko, A. V., Sargent, W. L. W., \& Peng, C. Y. 1997b, ApJS, 112,391

Ho, L. C., Shields, J. C., \& Filippenko, A. V. 1993b, ApJ, 410, 567

Hopkins, A. M., et al. 2003, ApJ, 599, 971

Jackson, N., \& Browne, I. W. A. 1990, Nature, 343, 43

Jahnke, K., et al. 2004, ApJ, 614, 568

Kauffmann, G., et al. 2003, MNRAS, 346, 1055

Kauffmann, G., \& Haehnelt, M. G. 2000, MNRAS, 311, 576

Kennicutt, R. C. 1998, ARA\&A, 36, 189

Kewley, L. J., Dopita, M. A., Sutherland. R. S., Heisler, C. A., \& Trevena, J. 2001, ApJ, 556, 121

Kewley, L. J., Geller, M. J., \& Jansen, R. A. 2004, AJ, 127, 2002

Magorrian, J., et al. 1998, AJ, 115, 2285

Martini, P. 2004, in Carnegie Observatories Astrophysics Series, Vol. 1: Coevolution of Black Holes and Galaxies, ed. L. C. Ho (Cambridge: Cambridge Univ. Press), 170

Nagao, T., Murayama, T., \& Taniguchi, Y. 2001, ApJ, 546, 744

Osterbrock, D. E. 1989, Astrophysics of Gaseous Nebulae and Active Galactic Nuclei (Mill Valley, CA: Univ. Science Books)

Ptak, A., Zakamska, N. L., Strauss, M. A., Krolik, J. H., Heckman, T. M., Schneider, D. P., \& Brinkmann, J. 2006, ApJ, in press (astro-ph/0510204)

Robertson, B., Hernquist, L., Cox, T. J., Di Matteo, T., Hopkins, P. F., Martini, P., \& Springel, V. 2006, ApJ, submitted (astro-ph/0506038)

Sanders, D. B., Soifer, B. T., Elias, J. H., Madore, B. F., Matthews, K., Neugebauer, G., \& Scoville, N. Z. 1988, ApJ, 325, 74

Schlegel, D. J., Finkbeiner, D. P., \& Davis, M. 1998, ApJ, 500, 525

Scoville, N. Z., Frayer, D. T., Schinnerer, E., \& Christopher, M. 2003, ApJ, 585, L105

Silk, J., \& Rees, M. J. 1998, A\&A, 331, L1

Solomon, P. M., \& Sage, L. J. 1988, ApJ, 334, 613

Spergel, D. N., et al. 2003, ApJS, 148, 175

Storchi-Bergmann, T. 1991, MNRAS, 249, 404

Storchi-Bergmann, T., Schmitt, H. R., Calzetti, D., \& Kinney, A. L. 1998, AJ, 115,909

Stoughton, C., et al. 2002, AJ, 123, 485

Strauss, M. A., et al. 2002, AJ, 124, 1810

Vanden Berk, D. E., et al. 2001, AJ, 122, 549

Veilleux, S., \& Osterbrock, D. E. 1987, ApJS, 63, 295

York, D. G., et al. 2000, AJ, 120, 1579

Zakamska, N. L., et al. 2003, AJ, 126, 2125

. 2005, AJ, 129, 1212 